\begin{tabular}{|c|c|}
\hline \multirow{3}{*}{ 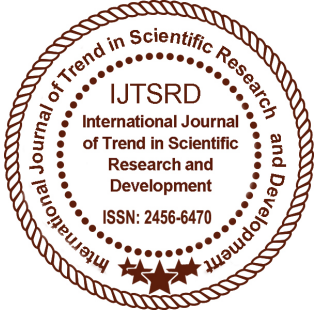 } & $\begin{array}{l}\text { International Journal of Trend in Scientific } \\
\text { Research and Development (IJTSRD) }\end{array}$ \\
\hline & International Open Access Journal \\
\hline & ISSN No: 2456 - 6470 | www.ijtsrd.com | Volume - 2 | Issue - 5 \\
\hline
\end{tabular}

\title{
Performance Analysis of Congestion Control Algorithm for Mobility Model in Mobile Ad-Hoc Networks by using Modified AODV Protocols
}

\author{
Kirtiraj Mohan Desai $^{1}$, Prof. Ms. T. T. Mohite Patil ${ }^{2}$ \\ ${ }^{1}$ ME Student, ${ }^{2}$ Professor \\ Sanjeevan Engineering \& Technology Institute, Panhala, Kolhapur, Maharashtra, India
}

\section{ABSTRACT}

The Mobile Ad hoc Networks (MANETs) often deal with obstacles that occur in the form of packet loss and so that can be efficiently reduced by involving congestion control which includes routing algorithm and a flow control at the network layer. We are proposing agent based agent based congestion control technique for MANETs in this paper with Modified AODV protocol. Here the mobile agents (MA) collect and broadcast the information about network congestion. The mobile agent based congestion control AODV routing protocol is used to avoid congestion in ad hoc network, and the modification done to have the same route for the MANET without changing it. Which can be done by inserting a protocol which alerts the sender to send some less packets in real time. The work of mobile agents is to carry routing information and nodes congestion status whenever the MANET is created. Whenever a mobile agent moves through the network, it selects a lessloaded neighbour node as its next hop and simultaneously keeps on updating routing table according to the node's congestion status. With the help of mobile agents, such is the role of MAs which creates the dynamic network topology in real time. Considering the simulation results using NS-2, we showed that our proposed technique efficient that traditional one which has numerous amount of packet loss. Our protocol has high delivery ratio and throughput with reduced delay which results in providing efficient protocol when compared with the different existing techniques.
KEYWORD: Congestion control Mobile Ad hoc Networks (MANETS), AODV routing protocol, Mobile Agents (MA), Total Congestion Metric (TCM), Transmission opportunity limit (TXOP).

\section{INTRODUCTION}

Mobile ad hoc networks have a wireless hosts whose main task is to communicate with each other when a fixed infrastructure is absent. The Route in between two mobile hosts in MANET consists of hops through other hosts in the network [4]. The most important task of finding and maintaining routes in MANET is nontrivial since host mobility causes frequent unpredictable dynamic topological changes in the network. Ad hoc mode is a method used for connecting the wireless devices to directly communicate with each other in dynamic fashion without any definite network infrastructure required. These are self-organizing, self-structuring networks. All nodes in the network do the work of both a participant and of establishing a short-lived network because as a router that has the functionality to forward the data. Ad hoc networking is same as peerto-peer networking and nodes move dynamically in network. Which means we don't require of any access point to connect to any other node in the network. [3] Mobile nodes without any problem can communicate directly through wireless link only if they are within each other's radio range and if they are not, then they rely on other neighbouring nodes which can act as routers to relay packets [2]. Mobile ad-hoc networks have the ability of dreaming connecting anywhere and at any time into reality. The ultimate goal of mobile ad hoc networking is to expansion of the mobility into 
the realm of mobile, wireless domains, which act as set a of nodes which can be combined both as router and as host means they form the network routing infrastructure in an ad-hoc fashion.

\section{CONGESTION DETECTION}

In this project paper we proposed a congestion control technique with modification in AODV routing protocol. AODV routing protocol is used to make a route from source to destination it avoids congestion in network by changing the path of flooded node. Single destination node is used to show congestion in multiple sources, which then results in path of formation of common nodes and congestion often occurs in that path.

\subsection{AODV:}

AODV protocol is an on demand protocol, in a network when any node wants to send a message to sink then it broadcasts request to all nearby nodes which is broadcasted further if no destination node is found. At the other end i.e the destination sends an acknowledgement message in form RREP is sent back following the same route from where it received route request from source and then source selects the path with least no of hops to send the message. Advantage of such approach is that the routing overhead is very much reduced. But disadvantage in such approach is that there is possibility of large delay from the moment the route is needed until route is actually acquired. Whenever a node receives such a message in the network and if it already has a route to the desired node, then it sends a message backwards via a temporary route to the requesting node. The necessitous node then starts using the route which closer w. r. $t$ no of hops through other nodes. Often some unused entries occurring in the routing tables are recycled after a particular time.

\subsection{Properties AODV Routing Protocol:}

$>$ Every node always assigns backup route(s) in alternative route table.

$>$ In the RREP phase Alternative route(s) determined.

$>$ At source node no complete route(s) information known.

$>$ There are no multiple complete routes available.

$>$ AODV fills up RREQs with unique IDs so duplication is avoided.

$>$ The backup route(s) or other routes which were discovered while route discovery are used whenever primary routes fail.
A particular information in the form of the packet format sent to neighbouring nodes which keep on adding their ID. And with help of this packet format information every node then follows the format and add its information to it and extract previous information to check whether they are supposed to receive that received packet or not.

\subsection{Congestion detection algorithm:}

Figure 1 given below shows the path shared by two sources to reach one common destination. We can see that at sharing of paths started node number 3 as indicated in the figure. Fixed Channel bandwidth can't be altered so it is mandatory to nodes that they have to transmit message within that given limit. So whenever a message from source 1 is has been transmitted to destination node and after some time source 2 transmits which has the same path as that of source 1 and no space to transmit the new message to destination. Then source 2 has to wait till the time there's some moment at node 3, and source 2 has to wait for a long time then source node may have some loss of packets as there's no feedback from sink node is received within expected time and again new packets will be lost if path is still occupied.

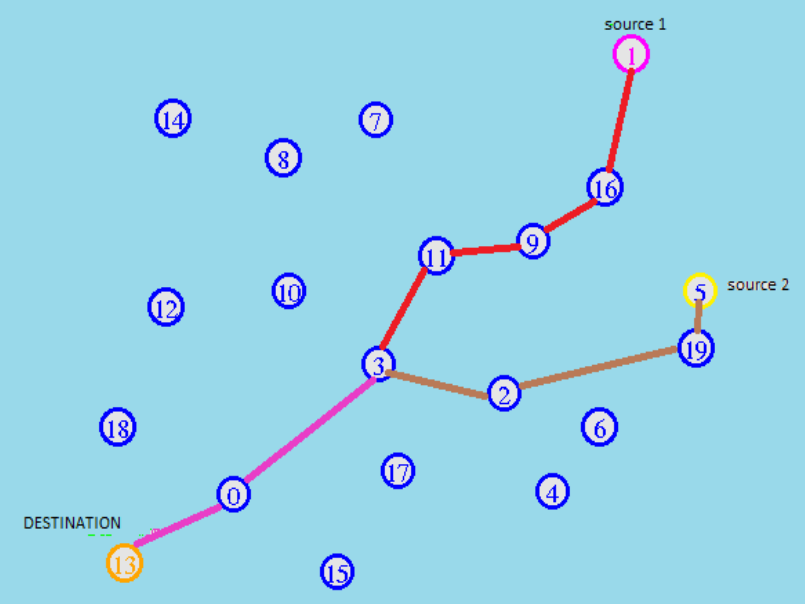

Figure 1: Congestion due to two sources sharing same path

Therefore the proposed algorithm works on particular common node, in above case at node 3 . The common node all the time will keep a track of bandwidth usage of channel, and as soon as it receives message from other source then it will check if there's space available in the channel, if there is available space so that it can transmit the partial packets or all packets, if it has then it will transmit or else it has to hold the message up to some specified time known as waiting time. 
Further when the problems occurs when the load on particular node heavy that it is losing packets due to overflow of buffer space available i.e. the threshold limit of the node. Then at such condition the that particular node will send message to source node to send less no packets at the rate which is currently it is sending, by doing so it will have time to send present packets in nodes and make available space for new ones. So the packet loss is avoided using this technique.

\section{PROPOSED WORK}

Figure 2 illustrates how the network performance with respect to function of the load. When MANET has less amount load on it, the throughput will linearly be proportional to the load and response time will also be almost unchanged. Whenever the load on the network reaches maximum limit of load handling capacity of the network capacity (threshold point), throughput wont' increase much with the load. Instead, due to load on network packets will be queued and the response time will become longer in this period causing delay. The throughput ratio will suddenly drop whenever packets don't flow through the nodes due to buffer overflow, which is called the cliff point as shown in Figure 2. we can observe congestion in many ways, for any interval of time, if there happens to be total sum of demands on a source is more than its available capacity, then source is said to be congested for the given instance interval of time. Mathematically speaking:

\section{$\Sigma$ Traffic Demand $>$ Resources Present}

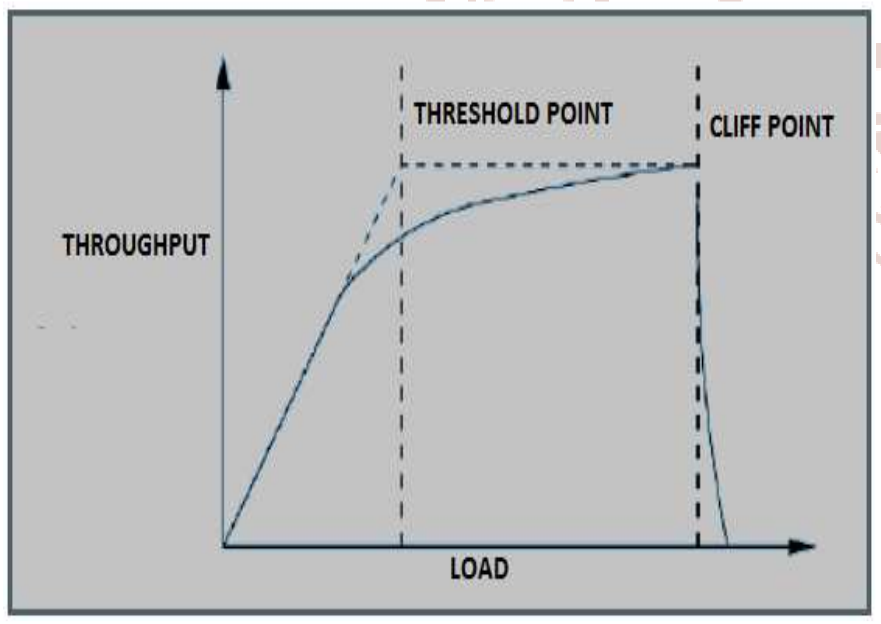

Figure 2: Effect of congestion on throughput

\section{PROPOSED CONGESTION CONTROL ALGORITHM:}

Below the proposed congestion control algorithm is explained with representation in the form of flowchart algorithm where we have used basic mobility models levy walk mobility model and the random walk mobility model. The packet drop ratioi. epdrand the threshold value for packet drop ratioi. epdrthr play an important role in analysing the MANET congestion problem. The average values for packet drop ratio are used to calculate the threshold values. The MANET network gets congested when values of pdr, delay goes on increasing above the threshold point and the value for throughput keeps on decreasing below threshold level, if nothing of this happens then we assume it to be a stable network. We need to calculate the buffer space if the network is congested, along with load and available channel capacity. The intermediate nodes sends a message to the source to send less number of packets and then it will calculate the congestion in the network only if these values are below working rate of network. And when the congestion control is achieved only then the process will stop. Now for the common path for two sources problem AODV uses the route request and route repeat request to confirm the route so that there is no overlapping of two routes between two sources and once route is confirmed, it constantly keeps sending message on the particular route until congestion occurs. In the figure 1 above we have the problem, Having two separate sources source 1 \& source 2 having a common destination to show congestion in the path occurring at node 3 . When such problem occur a new congestion free route is discovered by the source node which has been affected as given in figure 3 for source 2 . The newly discovered route doesn't have any affected nodes or nodes already serving some other route.

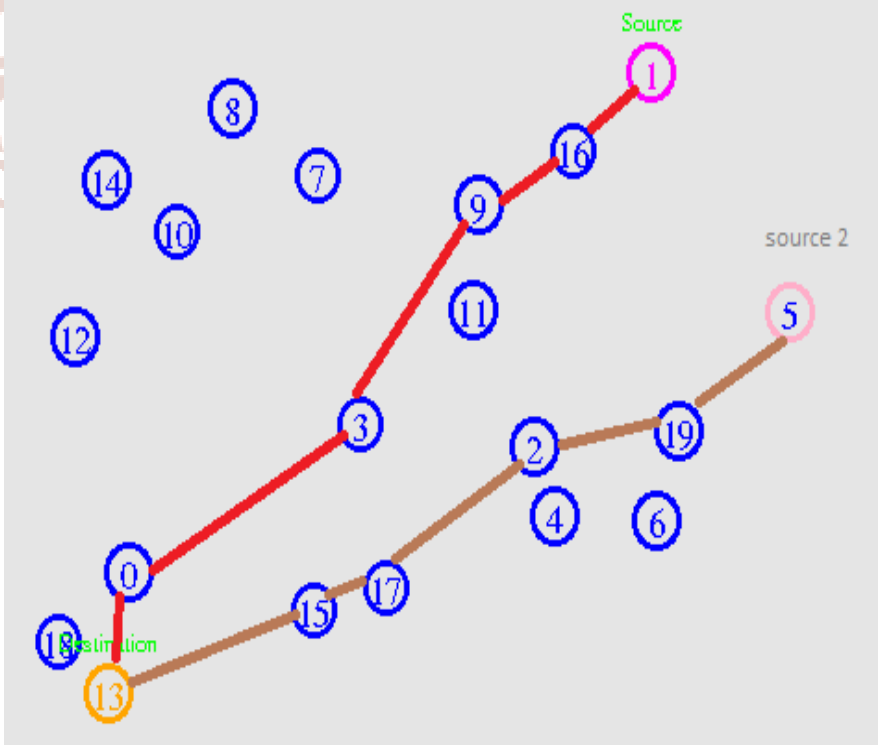

Figure 3: Discovering a congestion free route for the affected source for 20 nodes 
International Journal of Trend in Scientific Research and Development (IJTSRD) ISSN: 2456-6470

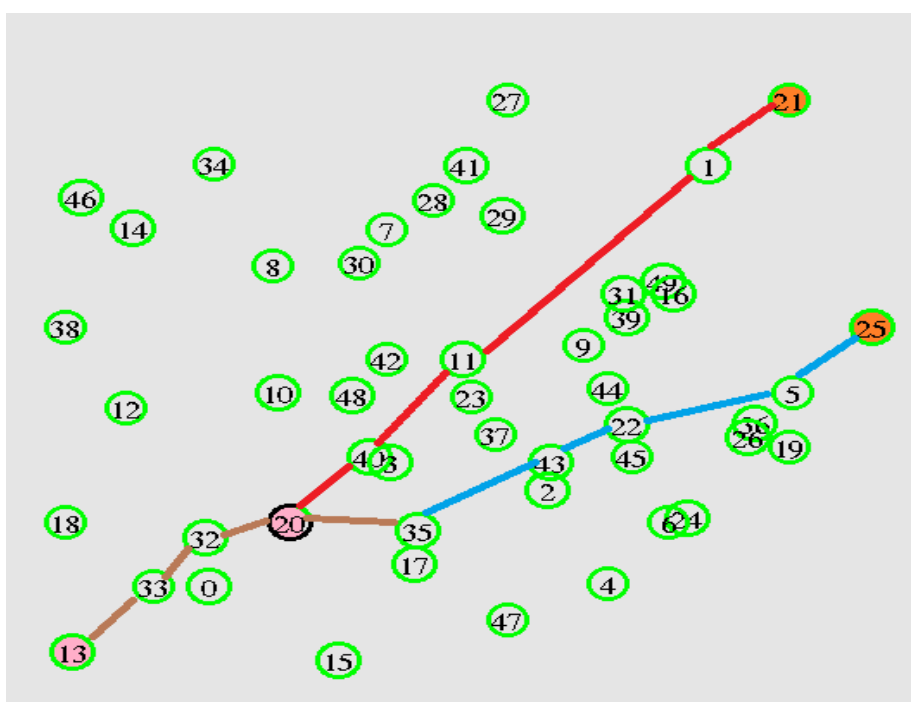

Figure 4: congestion due to common path between two sources in 50 nodes

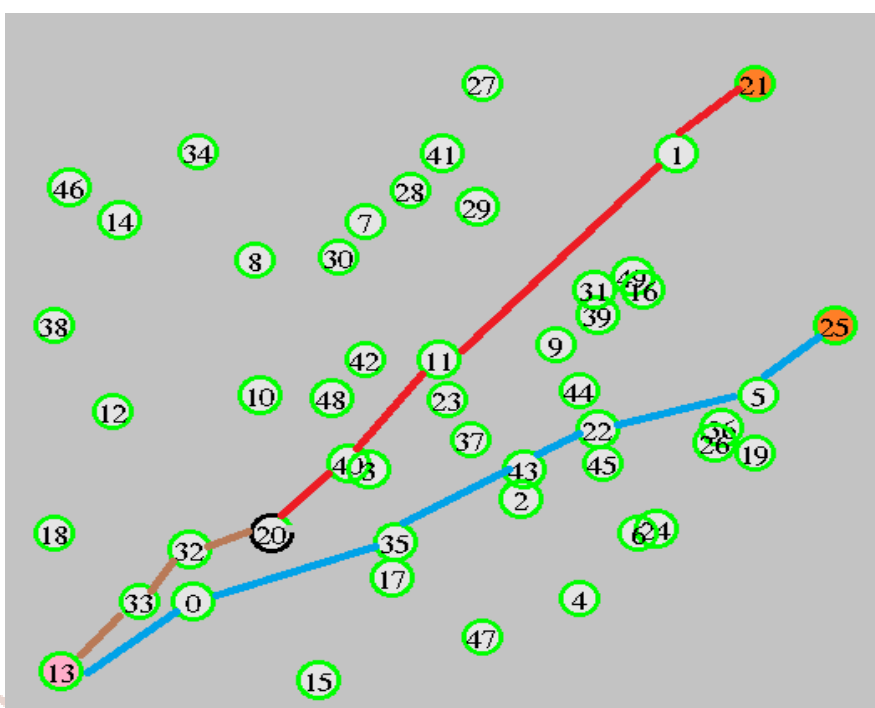

Figure 5: Discovering a congestion free route for the affected source for 50 nodes

\subsection{Flowchart of proposed Algorithm :}

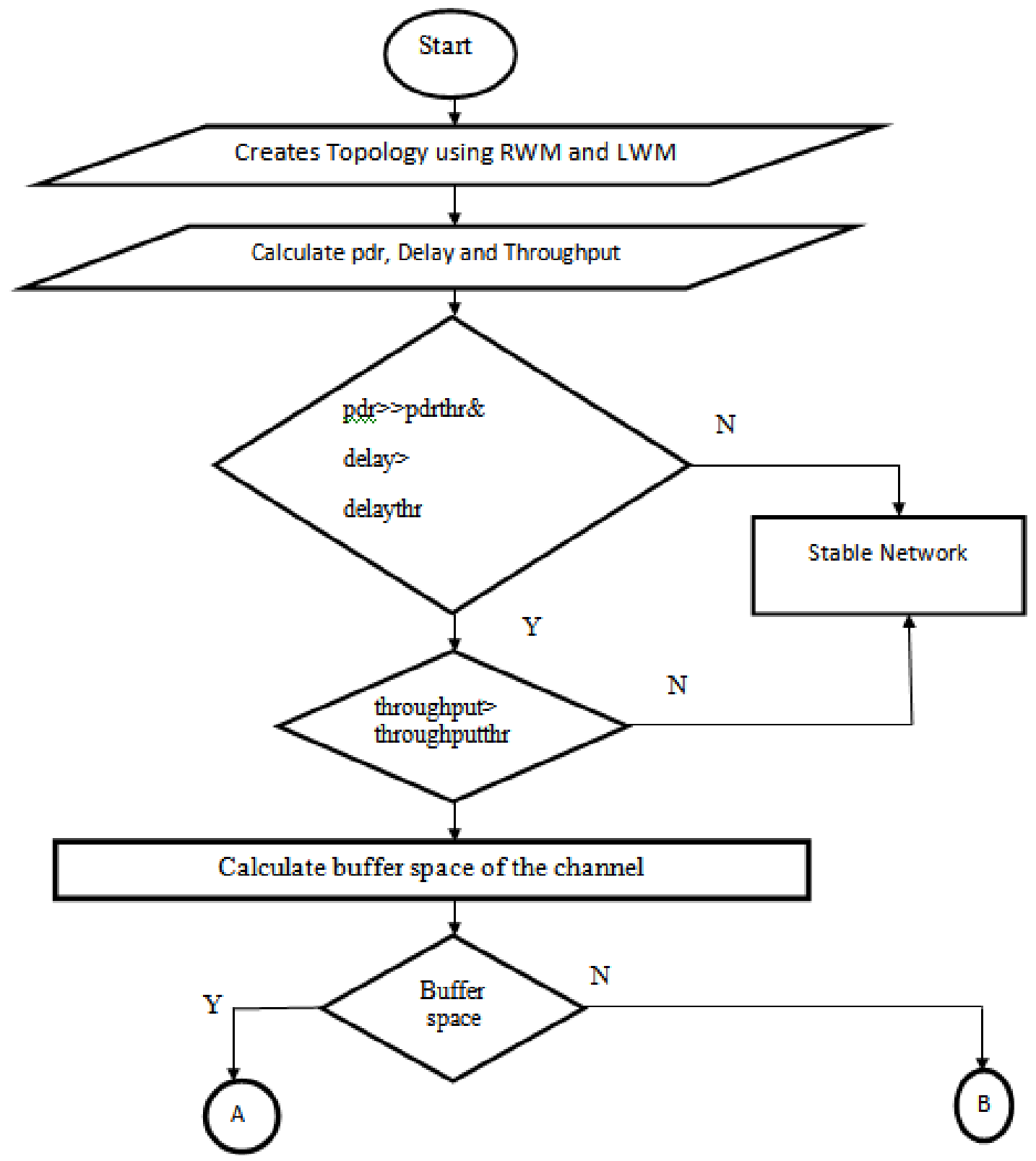




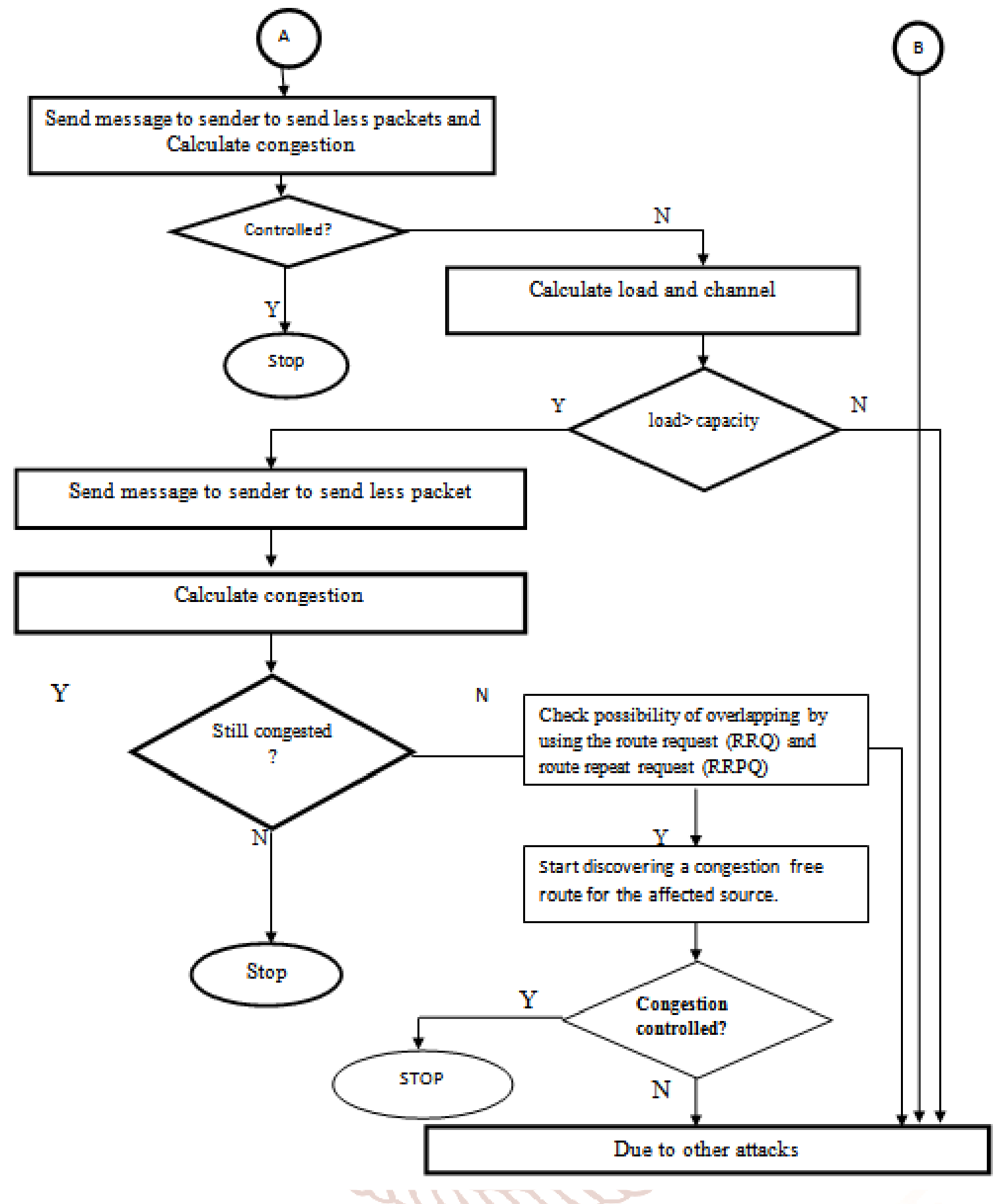

\section{CONCLUSION}

We started our study with analyzing various parameters causing congestion in MANET. After the analysis we further made study about how the congestion can be controlled and how the efficiency of the network can be improved. We came up with two possible methods which could cause problems in a network and finding solution to it. The congestion problem due to one common route to reach destination and the problem with the flow of the source which cause flooding a node due to which packets are lost. These two are important issues in a MANET and can be made congestion free using the algorithms.

\section{REFERENCES}

1. Bandana Bhatia, Neha Sood," AODV based Congestion Control Protocols: Review",(IJCSIT) International Journal of Computer Science and Information Technologies, Vol. 5 (3) , 2014, 4570-4575

2. L. Shrivastava, G. S. Tomar, and S. S. Bhadauria, "A Survey on Congestion Adaptive Routing Protocols for Mobile Ad-hoc Networks", Int. Journal of Computer Theory and Engineering, vol.3, Issue 2, 2011, pp. 189-196.

3. L. Xia, Z. Liu, Y. Chang, P. Sun, "An Improved AODV Routing Protocol Based on the Congestion 
Control and Routing Repair Mechanism", Int. Conf. Communications and Mobile Computing, IEEE, China, 2009, vol. 2, pp. 259-262.

4. Li, C. Dan, W. Min and L. Shurong, "Mobile Agent Based Congestion Control AODV Routing Protocol", Advanced Information Networking and Applications Workshops (WAINA), 2013 27th International Conference on, pp.62-66, 25-28 March 2013.

5. Barkha Shakya, Deepak Kulhare, Arpit Solanki, Investigation of TCP Congestion Control with Reliable Communication Techniques for MANET. International Journal of Computer Applications Vol65 No-14.Pp 0975-8887, 2013.

6. M. Ali, B. G. Stewart, A. Shahrabi and A. Vallavaraj, " Congestion Adaptive Multipath Routing for Load Balancing in Mobile Adhoc
Networks", Innovations in Information Technology (IIT), 2012 International Conference on , pp.305-309, 18-20 March 2012.

7. Pingale, A Rakshe, S. A. Jain, and S. R. Kokate, "A Study of congestion aware adaptive routing protocols in MANET", International Journal of Advanced Technology and Engineering Research (IJATER), May 2012, v. 2, Issue 2.

8. S. Floyd and K. Fall, "Promoting the use of endto-end congestion control in the internet" IEEE/ACM Transactions on Networking, 7(4), August 1999.

9. Nishu Garg, R. P. Mahapatra "MANET Security Issues", IJCSNS International Journal of Computer Science and Network Security, VOL.9 No.8, August 2009. 